\title{
Understanding sawtooth activity during intense electron cyclotron heating experiments on TCV
}

\author{
I. Furno, C. Angioni, F. Porcellia , H. Weisen, R. Behn, T.P. Goodman, \\ M.A. Henderson, Z.A. Pietrzyk, A. Pochelon, H. Reimerdes, E. Rossi ${ }^{b}$ \\ Centre de Recherches en Physique des Plasmas, Association Euratom-Confédération Suisse, \\ Ecole Polytechnique Fédérale de Lausanne, Lausanne, \\ Switzerland \\ ${ }^{a}$ Istituto Nazionale Fisica della Materia and Dipartimento di Energetica, \\ Politecnico di Torino, Turin, Italy \\ b Institute for Fusion Studies, Department of Physics, The University of Texas at Austin, \\ Austin, Texas, United States of America
}

\begin{abstract}
Different types of central relaxation oscillations are observed in the presence of ECH depending on the location of the deposited power. In the TCV tokamak, normal sawteeth, i.e. triangular sawteeth similar to ohmic sawteeth, and saturated sawteeth are observed with central ECH power deposition, while giant sawteeth and 'humpback' oscillations occur when heating close to the sawtooth inversion surface of the local soft $\mathrm{X}$ ray emissivity. New measurements with high temporal resolution show that the crash phase of these sawtooth types is accompanied by a reconnection process associated with an $m / n=1$ resistive internal kink mode. After the sawtooth crash, full magnetic reconnection is observed in normal and in saturated sawteeth, while for giant and humpback sawteeth the reconnection process is incomplete and poloidally asymmetric temperature profiles persist after the crash. The detailed dynamics of the magnetic island associated with the resistive internal kink mode are described by a displacement function which is inferred from the experimental data. In normal sawteeth, the kink mode is destabilized just before the crash, while in all other sawtooth types a magnetic island exists for a significant fraction of the sawtooth period. The different types of sawteeth have been simulated using a numerical code based on a theoretical model which describes the evolution of the electron temperature in the presence of localized heat sources and of a magnetic $m / n=1 / 1$ island.
\end{abstract}

\section{Introduction}

Sawtooth oscillations in tokamak discharges are periodic relaxations of the central electron temperature and density which develop when the safety factor on-axis $q_{0}$ drops below unity. A slow rise (sawtooth ramp) of these plasma parameters in the central region of the discharge, determined by heat deposition and transport, is followed by a rapid drop (sawtooth crash) resulting in an expulsion of energy and particles from the plasma core. Although these internal instabilities have long been observed and studied in all tokamaks, the understanding of the underlying physical mechanism is incomplete. Furthermore, recent experiments have revealed peculiar new plasma features when localized $\mathrm{ECH}$ is applied in sawtoothing tokamak discharges. Examples are the observation on the RTP tokamak [1] of sharp temperature gradients just outside the sawtooth inversion radius and the multipeaked temperature profiles observed in the TEXT Upgrade [2] and RTP [1] experiments. On TCV [3], the sawtooth activity is strongly dependent on the ECH deposition conditions. In Ref. [4], a classification of the different types of observed sawteeth was proposed, from the usual triangular sawteeth, obtained with on-axis power deposition, to non-standard behaviour when the location of the resonance position is moved off-axis. In particular, when the ECH power is deposited close to the $q=1$ surface, the line integrated soft X ray traces can exhibit 'humpback'like features. These internal relaxation oscillations, which were first observed with central electron cyclotron counter-current drive [5] in the T-10 tokamak, can also occur under similar conditions on TCV [6], although current drive is not essential.

In previous TCV papers on this subject $[4,7,8]$, an understanding of the sawtooth dynamics was hampered by the limited temporal resolution of the available diagnostics which did not allow us to resolve 


\section{Furno et al.}

the sawtooth crash phase. This has now been overcome by an improvement of the time resolution of the TCV soft X ray tomographic system [9], which allows monitoring of the sawtooth crash $\sim 10$ times faster than the crash time, which is typically $\sim 100 \mu \mathrm{s}$.

In this article, we report on these high temporal resolution measurements of the sawtooth oscillations during ECH. An interpretation of the observed phenomena is provided and linked to a recently developed theoretical model [10]. The experimental features during the different types of sawteeth are reproduced by a numerical code which is based on this theoretical model. It is shown that the sawtooth behaviour is the consequence of specific features in the electron temperature profiles produced by both $\mathrm{ECH}$ and the advection and mixing of electron thermal energy associated with an $m / n=1 / 1$ resistive internal kink mode [11]. Possible explanations of the remaining inconsistencies are proposed, showing that a single model can explain the variety of different sawteeth.

The remainder of the article is organized as follows. In Section 2, the TCV tokamak, the ECH system and the relevant diagnostics are briefly reviewed. In Section 3, the experimental results and a heuristic interpretation are presented. A theoretical model is derived in Section 4 and the simulations of the different types of sawteeth are discussed in Section 5. Finally, the conclusions are summarized in Section 6.

\section{Experimental set-up}

TCV is a tokamak with major radius $R=0.88 \mathrm{~m}$, minor radius $a=0.25 \mathrm{~m}$, vacuum vessel elongation $\kappa=3$ and vacuum central magnetic field $B \leq$ $1.54 \mathrm{~T}$. For these experiments, TCV was operated with three $82.7 \mathrm{GHz}, 500 \mathrm{~kW}$ gyrotrons, with a $2 \mathrm{~s}$ pulse length, for heating at the second cyclotron harmonic resonance using the extraordinary mode. The three launching mirrors are separately orientable in the toroidal and poloidal directions. The vertical microwave beam width near the plasma centre is $w=2.5 \mathrm{~cm}$ in free space (beam intensity $\propto \exp \left[-2(l / w)^{2}\right]$, where $l$ is the distance transverse to the direction of the beam propagation). Local ECH flux surface averaged power densities in excess of $10 \mathrm{MW} / \mathrm{m}^{3}$ can be obtained.

The soft X ray tomography system [9] consists of ten pinhole cameras at the same toroidal location, each equipped with a $47 \mu \mathrm{m}$ beryllium filter and a linear array of 20 silicon photodiodes, which are sensitive to electromagnetic radiation in the range 1-10 keV. The system has been recently upgraded with a 16 bit data acquisition module with a $13 \mu \mathrm{s}$ sampling rate, allowing high time resolution measurements of the sawtooth crash dynamics. The local soft $\mathrm{X}$ ray emissivity distribution is reconstructed from line integrated measurements by means of the minimum Fisher information regularization method [9] on a grid of square pixels (of side $3 \mathrm{~cm}$ ). The time sequences of the reconstructed emissivities $S_{X}(r, t)$ are analysed using the singular value decomposition (SVD) method [12] to obtain the spatial structures $u_{k}$ (topos) and their corresponding temporal evolution $v_{k}$ (chronos) such that

$S_{X}(r, t)=\sum u_{k}(r) v_{k}(t) s_{k}$

where $s_{k}$ are the singular values. Structures with poloidal mode numbers up to $m=3$ are resolved by the SVD, and coherent rotating structures such as MHD modes can be identified. It has been shown $[4,13,14]$ that there is a strong correlation between the sawtooth shape and the heating location relative to the sawtooth inversion surface, in the sense that small changes in the location of the ECH deposition with respect to the inversion surface lead to large changes in sawtooth period, amplitude and MHD activity. The SVD method also provides a reliable tool in the determination of the sawtooth inversion surface of the local soft $\mathrm{X}$ ray emissivity. In the following, the sawtooth inversion contours are defined by the equation

$\hat{S}_{X}\left(r, t_{\text {before }}\right)-\hat{S}_{X}\left(r, t_{\text {after }}\right)=0$.

Here, $\hat{S}_{X}$ is obtained from the SVD expansion by discarding topo/chrono couples which are not poloidally symmetric, i.e. corresponding to poloidal mode numbers $m \geq 1$, and the times $t_{\text {before }}$ and $t_{\text {after }}$ are relative to the sawtooth crash time. The effective inversion radius is defined as $r_{i n v}=\sqrt{A_{i n v} / \pi}$, where $A_{i n v}$ is the cross-sectional area within the inversion contour defined by Eq. (1). The size of the grid used in the tomographic reconstruction is taken as an estimate of the uncertainty in $r_{i n v}$.

The radial displacement $\xi(t)$ of the position of the maximum emissivity associated with the kink instability during the sawtooth crash is also extracted from the reconstructed soft $\mathrm{X}$ ray emissivities. The time evolution of $\xi(t)$ is assumed to represent the displacement of the magnetic axis, which is not predicted theoretically by the model to be described in 
Section 4. In what follows, we define the effective displacement

$\xi(t)=\sqrt{\kappa_{0}\left(\left(R-R_{0}\right)^{2}+\frac{\left(Z-Z_{0}\right)^{2}}{\kappa_{0}^{2}}\right)}$

where $(R, Z)$ is the position of maximum emissivity within the hot core, $\left(R_{0}, Z_{0}\right)$ refers to the same point before the instability develops and $\kappa_{0}$ is the elongation at the plasma centre. From this definition, $\xi(t)$ is constant on flux surfaces and therefore can be directly compared with the effective inversion radius $r_{i n v}$. The elongation of the magnetic flux surfaces varies little inside the $q=1$ surface, which justifies the use of the central elongation in Eq. (2).

The soft $\mathrm{X}$ ray emission is also monitored by four toroidally equispaced silicon photodiodes $(50 \mu \mathrm{m}$ beryllium filter, $250 \mathrm{kHz}$ acquisition frequency) placed at the top of the vessel and viewing the plasma on chords intersecting the poloidal midplane at a distance about $7 \mathrm{~cm}$ from the centre of the vessel. These are used to determine toroidal mode numbers $n=1$, 2 , assuming there is no aliasing from toroidal mode numbers $n \geq 3$.

Other diagnostics available on TCV include toroidal and poloidal arrays of Mirnov coils, a 14 channel far infrared interferometer (FIR) and a 25 point multipulse Thomson scattering system [15]. The latter provides profiles of the electron temperature and density with a spatial resolution of $40 \mathrm{~mm}$ in the vertical direction and $3 \mathrm{~mm}$ in the radial and toroidal directions with a typical sampling rate of $60 \mathrm{~Hz}$. Using a combination of three almost collinear laser beams, sampling intervals down to $0.4 \mathrm{~ms}$ can be achieved in the so-called burst mode, when the three lasers are triggered close together.

The position of the ECH absorption region and the total absorbed power are determined by the ray tracing code TORAY [16].

\section{Experimental observations and heuristic understanding}

In this section, we report on high temporal resolution measurements of the various types of relaxation oscillation under localized and intense ECH. The injected power $P_{E C H}$ exceeds the power in the ohmic target by up to a factor of 4 and the microwave beam is launched perpendicular to the toroidal magnetic field. In this geometry, a small amount of current (typically less than $2 \%$ of the inductive plasma current) is driven by the microwave beam due to the

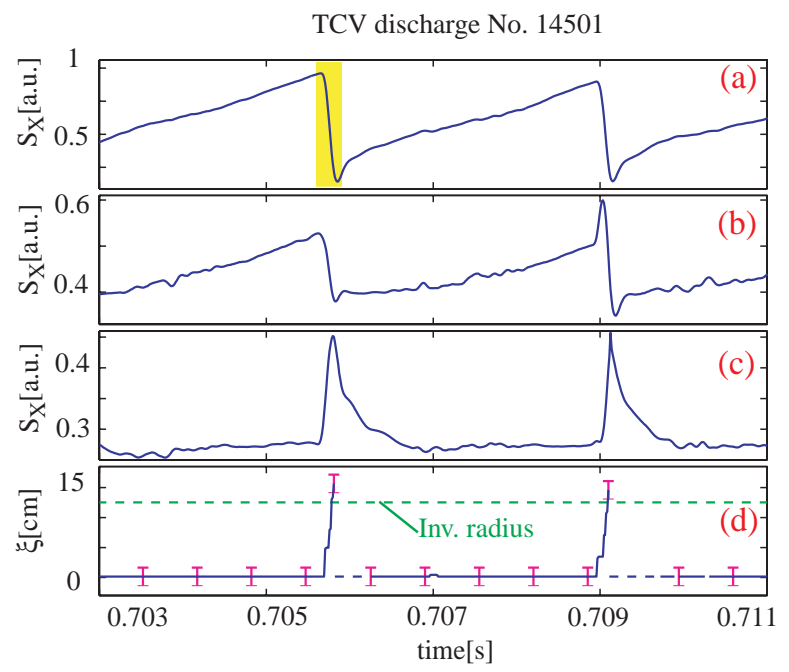

Figure 1. Evolution of the soft $\mathrm{X}$ ray emissivity at different radial positions $r$ during triangular sawteeth in TCV discharge 14501: (a) on-axis, $r=0$; (b) just inside the inversion radius, $r=0.82 r_{i n v}$; (c) just outside the inversion radius, $r=1.21 r_{i n v}$; (d) the hot core radial displacement $\xi(t)$ (blue line) together with the sawtooth inversion radius (green dashed line). The position of the hot core is not shown immediately after the sawtooth collapse since it is not well defined.

pitch of the magnetic field lines when the ECH power is deposited off-axis. Although it has recently been shown that the sawtooth period is strongly sensitive to small amounts of current driven close to the $q=1$ surface [17], this turns out not to be the case for the sawtooth shape, which is seen not to vary dramatically as a consequence of small variations of the driven current.

Standard and saturated sawteeth, related to central heating, together with large, almost triangular sawtooth and humpback oscillations, which occur with ECH power deposited close to the sawtooth inversion surface, are analysed.

\subsection{Standard sawteeth}

An example of a standard triangular sawtooth obtained with on-axis ECH power deposition is shown in Fig. 1. In what follows, the plasma parameters are referred to the LCFS and in the present case are $\delta=0.42, \kappa=1.41, I_{p}=400 \mathrm{kA}$ and $P_{E C H}=900 \mathrm{~kW}$. The sawtooth period $\tau_{S T}$ is $\approx 3.4 \mathrm{~ms}$, the electron energy confinement time $\tau_{E}$ is $3.3 \mathrm{~ms}$ and the resistive diffusion time $\tau_{\eta}$ can be estimated to be $\approx 450 \mathrm{~ms}$. The relative crash amplitude is $\Delta S_{X}(0) / \bar{S}_{X}(0) \approx 0.65$, where the bar indicates the 


\section{Furno et al.}

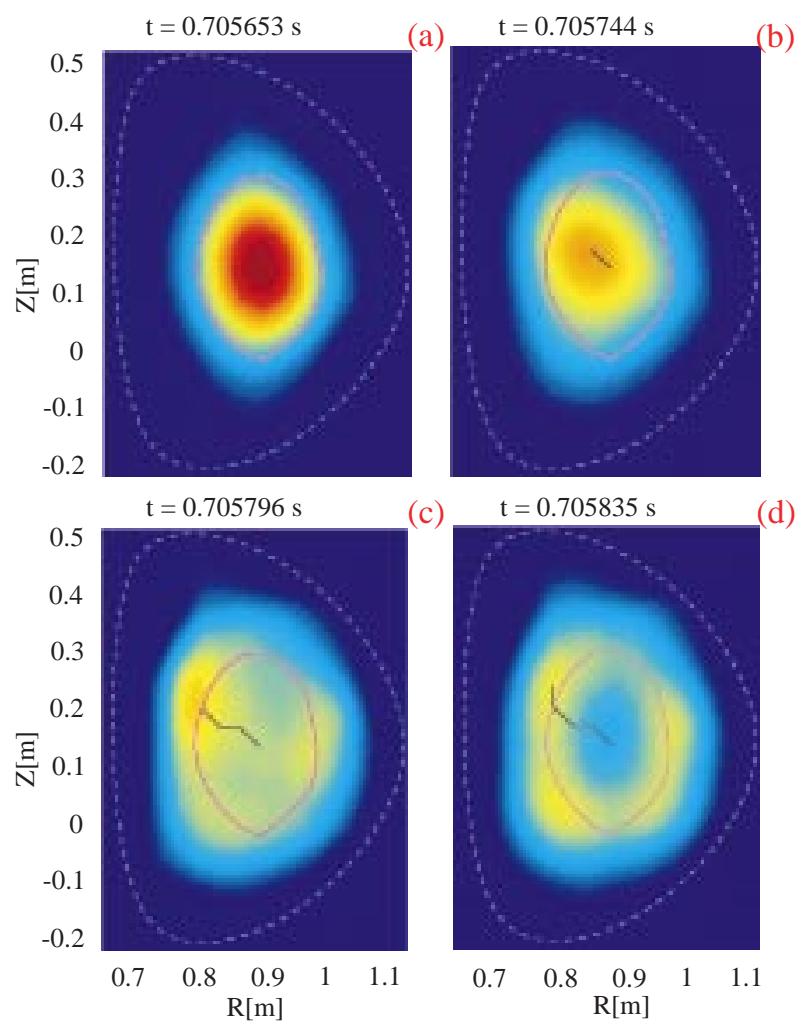

Figure 2. A series of tomographic reconstructions of the soft $\mathrm{X}$ ray emissivity, during the phase of the sawtooth crash highlighted in yellow in Fig. 1. The hot core displacement (b, c) and the formation of the hot ring (d) are shown. The units are normalized and the colour scale is such that red and blue correspond, respectively, to high and low emissivities. The LCFS is shown as a white dashed line and the sawtooth inversion surface as a magenta line. The superimposed black line indicates the hot core trajectory from its position before the instability develops to the position corresponding to the time at each frame.

average of a quantity evaluated before and after the sawtooth crash. The temporal evolution of the local emissivities is shown in Figs $1(\mathrm{a}-\mathrm{c})$ for three different radial positions together with the radial displacement $\xi(t)$ of the hot core (the region of high emissivity) in Fig. 1(d). In Fig. 2, the soft $\mathrm{X}$ ray emissivity distribution is shown at different times during the sawtooth collapse together with the LCFS, the sawtooth inversion radius, as calculated from soft $\mathrm{X}$ ray tomography, and the hot core trajectory.

The soft $\mathrm{X}$ ray emissivity is poloidally symmetric, Fig. 2(a), just before the crash, with no detectable precursor oscillations. At the sawtooth crash, an $m / n=1 / 1$ instability grows and causes the hot core

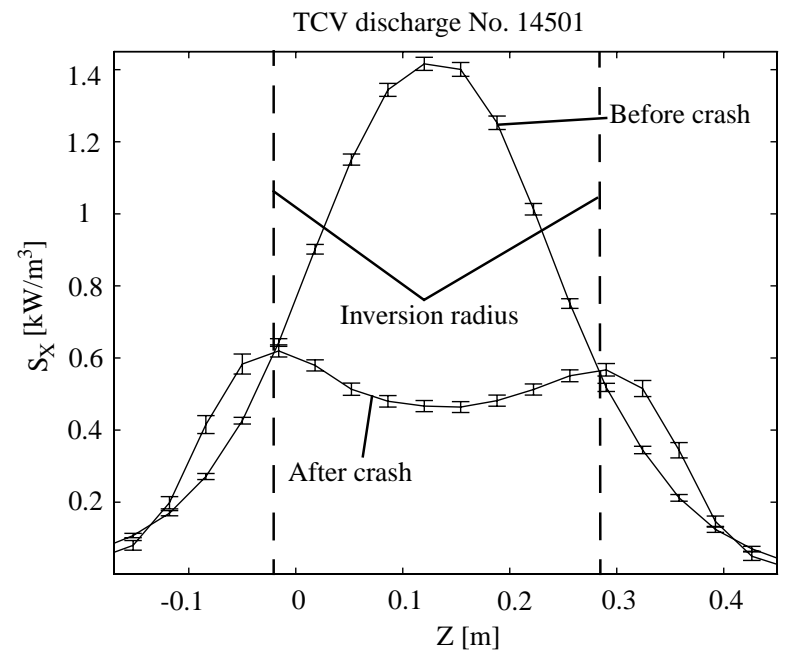

Figure 3. Soft X ray emissivity profiles along a central vertical cut showing the result of a crash for a triangular sawtooth with on-axis ECH power deposition. The position of the sawtooth inversion radius is indicated by the dashed lines.

to move outwards in minor radius as a nearly rigid structure in $\approx 120 \mu \mathrm{s}$. The hot core does not spiral in the poloidal plane as one would expect for a toroidally rotating plasma. The rapidity of the hot core displacement during the sawtooth crash $(\approx 120 \mu \mathrm{s})$ compared with the frequencies of the modes usually observed on TCV, which are between 3 and $6 \mathrm{kHz}$, suggests a minimum poloidal rotation of $\approx 120^{\circ}$, which is not observed in Fig. 2. At present, this discrepancy is not understood.

The final radial shift of the hot core from the initial position is $\xi_{\max } \approx 15 \mathrm{~cm}$. During the displacement, the hot core intensity diminishes, Fig. 2(b), and the expelled heat redistributes on a hot ring localized, within the error bars, at the sawtooth inversion radius $r_{i n v} \approx 12 \mathrm{~cm}$, Fig. $2(\mathrm{c})$. The soft $\mathrm{X}$ ray emissivity profile is poloidally symmetric at the end of the sawtooth crash, Fig. 2(d). The heat redistribution on the annular ring, probably due to heat transport along magnetic field lines, appears to be much faster than the hot core radial displacement and cannot be resolved with the $13 \mu$ s time resolution of the tomographic system. Small regions of locally lower or higher emissivity at the inversion surface, visible in Fig. 2(d), can be explained by artefacts of the tomographic reconstruction technique.

The effect of the sawtooth oscillation on the soft $\mathrm{X}$ ray emissivity profiles is shown in more detail in Fig. 3. The peaked pre-crash profile evolves into a hollow post-crash profile, with 
$S_{X}\left(r_{i n v}\right) / S_{X}(0) \approx 1.35$. The timescale of the evolution of the post-crash profile depends on both the heating conditions and plasma parameters. In the present case, the hot ring is visible for $\approx 150 \mu$ s then the profile gradually becomes more peaked as the plasma is heated until the next sawtooth collapse.

In order to quantify the uncertainties due to random errors, a set of 100 tomographic reconstructions were calculated from the line integrated data adding, to all channels, a normally distributed random noise with a mean value of zero and a standard deviation of $3 \%$ of the signal amplitude, which is worse than the experimental measurements imply [9]. The error bars shown in Fig. 3 are the standard deviations of the local emissivities evaluated for this set of reconstructions. Systematic errors due to uncertainties in the position of the lines of sight of the tomographic system and in the efficiency of the silicon photodiodes are taken into account by the procedure described in Refs $[9,18]$. Sufficiently large statistical errors were chosen and weighted with the inverse of the étendue for each line of sight, since a small étendue implies a large angle of incidence and thus a larger uncertainty on the value of the étendue and the incorrectly estimated photodiode efficiency. Another systematic error comes from the tomographic inversion method itself. Simulated line integrated data from hollow soft X ray emissivity profiles were tomographically inverted using the minimum Fisher regularization method. The reconstructed profiles are seen to underestimate the initial $\left[S_{X}\left(r_{i n v}\right)-S_{X}(0)\right] / S_{X}(0)$ ratio by about a factor of two. We can thus conclude that a hot ring does indeed exist for which our inversion technique provides only a lower limit for the hollowness.

Interpretation of the variation in the soft $\mathrm{X}$ ray emissivity in terms of changes in the electron temperature is not straightforward. Although the Thomson scattering system provides direct measurements of the $T_{e}$ and $n_{e}$ profiles, no measurement of the $T_{e}$ profiles during the short lifetime of the hot ring have been obtained so far. This is due to the limited repetition rate and the difficulty of synchronizing the Thomson scattering laser pulses with the sawtooth crash. A rough estimate of the hollowness of the electron temperature profile can be obtained from the soft X ray measurements, assuming $\Delta T_{e} / T_{e}=$ $\gamma \Delta S_{X} / S_{X}$, where $\gamma=d\left(\ln S_{X}\right) / d\left(\ln T_{e 0}\right)$ is the factor describing the temperature dependence of the soft $\mathrm{X}$ ray emissivity of the dominant impurity, carbon, in TCV [19]. For the case shown in Fig. 3, the estimate gives $T_{e}\left(r_{i n v}\right) / T_{e 0} \approx 1.2$, assuming flat $n_{e}$ and $Z_{\text {eff }}$ profiles. Abel inverted interferometric measurements show that particles are expelled from the plasma centre $\left(\Delta n_{e}(0) / \bar{n}_{e}(0) \approx 0.09\right)$ during the sawtooth crash, resulting in a flattening of the $n_{e}$ profile with $n_{e}\left(r_{i n v}\right) / n_{e}(0)$ below the error bars $(5 \%)$ of the inversion technique. Since there is no independent measurement of the $Z_{\text {eff }}$ profile during the lifetime of the hot ring, we cannot exclude the possibility that a local enhancement of $Z_{\text {eff }}$ at the hot ring position may contribute to the soft $\mathrm{X}$ ray intensity. Such an enhancement could result from a highly peaked $Z_{\text {eff }}$ profile in the plasma core before the sawtooth crash. The $Z_{\text {eff }}$ profile can be obtained from simultaneous soft $\mathrm{X}$ ray, $T_{e}$ and $n_{e}$ measurements in the manner proposed in Ref. [20]. This has been done for the present case and pre-crash $Z_{\text {eff }}$ profiles which are flat inside the inversion radius were obtained. This result does not support the evidence for the existence of impurity accumulation and hence makes it implausible that the hot ring emissivity is due to an enhanced impurity density inside the ring.

In Section 5, it will be shown that the formation of the hot ring can be explained as a consequence of the advection and mixing of the electron thermal energy during the growth of the $m / n=1 / 1$ magnetic island. However, a hot ring forms only when the pre-crash temperature profile is sufficiently peaked, as in the case for on-axis ECH deposition. With ohmic heating only, this condition is normally not fulfilled and the hot ring is not observed.

The absence of post-cursor oscillations on both Mirnov coils and soft X ray traces suggests a full reconnection process, in the sense that poloidal magnetic symmetry is restored at the end of the sawtooth crash. This requires that the original magnetic axis moves up to the mixing radius $r_{\text {mix }}$ at the end of the reconnection process, i.e. $\xi_{\max } \cong r_{\operatorname{mix}}$ (see Section 4 for the mathematical definition of $r_{m i x}$ ). This radius is slightly larger than the sawtooth inversion radius, by an amount which depends on the safety factor and electron temperature profiles before the crash (see the Appendix in Ref. [4]). The maximum value of the hot core displacement, $\xi_{\max } \approx 15 \mathrm{~cm}$, compared with $r_{i n v} \approx 12 \mathrm{~cm}$, is consistent with this interpretation.

\subsection{Saturated sawteeth}

Tomographic reconstructions of a saturated sawtooth are shown in the second example from TCV discharge 14385 with central ECH. The plasma 


\section{Furno et al.}

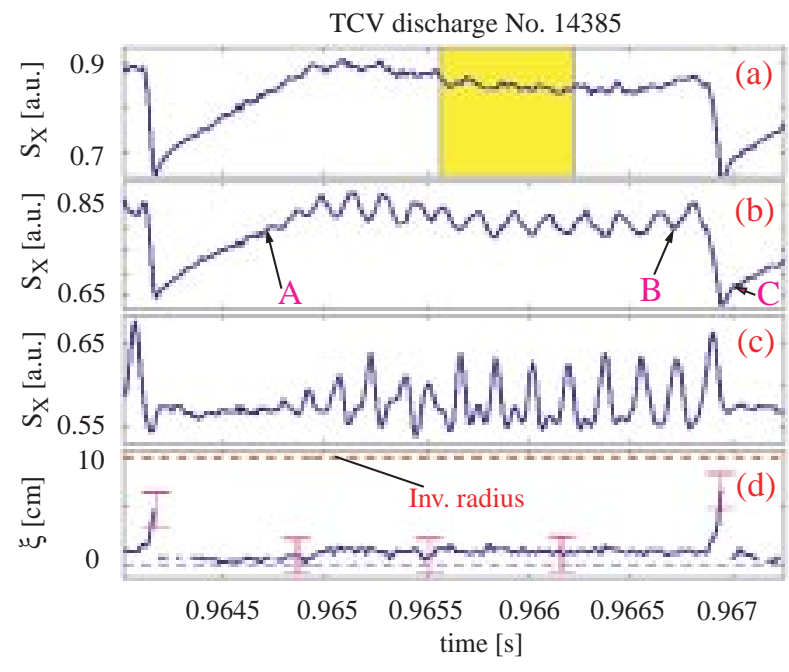

Figure 4. Time evolution of the soft $\mathrm{X}$ ray emissivity at different radial positions $r$ during a saturated sawtooth in TCV discharge 14385: (a) close to the magnetic axis, $r=0.02 r_{i n v}$; (b) slightly off-axis, $r=0.28 r_{i n v}$; (c) just inside the inversion radius, $r=0.9 r_{i n v}$, where the frequency doubling is clearly visible; (d) the hot core radial displacement $\xi(t)$ (blue line) together with the sawtooth inversion radius (red dashed line). The position of the hot core is not shown immediately after the sawtooth collapse since it is not well defined.

parameters are $\delta=0.37, \kappa=1.46, I_{p}=266 \mathrm{kA}$, $P_{E C H}=850 \mathrm{~kW}, \tau_{E}=3.6 \mathrm{~ms}$ and $\tau_{\eta} \approx 250 \mathrm{~ms}$. The ECH resonance position is located on the magnetic axis with a radial power deposition width $\delta_{a b s} \approx 6 \mathrm{~cm}$. This discharge has a lower current than that of the previous case with a consequently smaller inversion radius of $r_{i n v} \approx 9 \mathrm{~cm}$. In Fig. 4, the temporal evolution of the tomographically inverted soft $\mathrm{X}$ ray emissivity at different radial positions is shown together with the hot core radial displacement $\xi(t)$.

The sawtooth crash is followed by a reheating phase, in which the soft $\mathrm{X}$ ray emissivity profile peaks, conserving the poloidal symmetry. This phase is followed for $t>0.965 \mathrm{~s}$ by a saturated phase during which the central soft $\mathrm{X}$ ray emissivity remains almost constant or even decreases until the following sawtooth crash. The saturated phase is accompanied by strong mode activity, resulting in off-axis oscillations, Fig. 4(b). The frequency of the oscillations (in the present case $\approx 6 \mathrm{kHz}$ ) can vary between 3 and $7 \mathrm{kHz}$, depending on ECH and plasma conditions. Close to the inversion radius, Fig. 4(d), a frequency doubling is sometimes observed. During

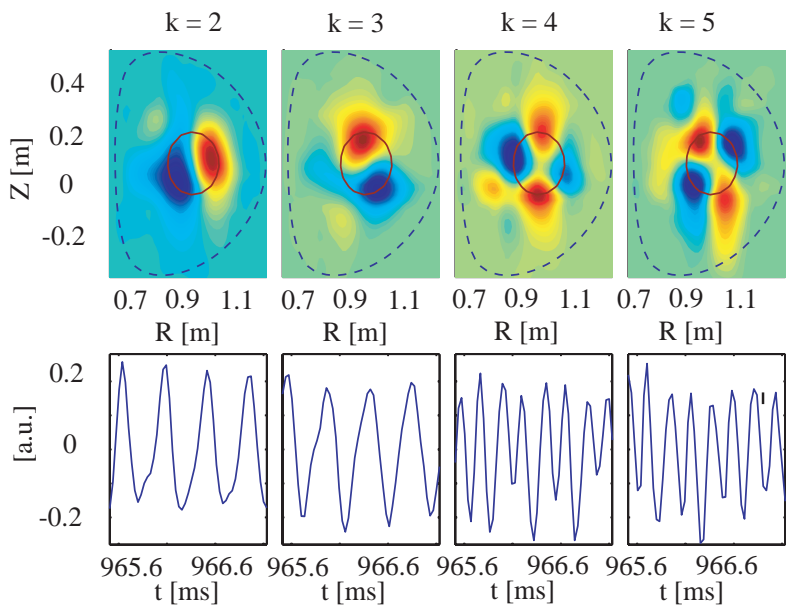

Figure 5. SVD analysis of the reconstructed soft $\mathrm{X}$ ray emissivity distribution during the saturated phase of the sawtooth, highlighted in yellow in Fig. 4. Shown are the topo/chrono pairs corresponding to the second to fifth largest eigenvalues, $S_{k=2,3,4,5}$. The top row shows the spatial eigenmodes (topos) with the LCFS (dashed black line) and the sawtooth inversion radius (red line). The corresponding temporal eigenvectors (chronos) are shown in the bottom row.

the saturated phase, the plasma hot core appears to rotate around the position it had before mode onset at a distance $\xi \approx 1 \mathrm{~cm}$.

In Fig. 5, the mode structure of the soft $\mathrm{X}$ ray emissivity, from SVD analysis, is shown in a small time window during the saturated phase, highlighted in yellow in Fig. 4(a). The first topo/chrono pair, corresponding to the largest singular value, is not shown because it describes the temporal evolution of the average emissivity profile and thus is not relevant to the mode identification. Inspection of Fig. 5 reveals the presence of two rotating modes, each consisting of two oscillating topo/chrono pairs in quadrature (i.e. both topo and chrono are phase shifted by $\pi / 2$ ) and having poloidal mode numbers $m=1$ (topo/chrono pairs $k=2,3$ ) and $m=2$ (topo/chrono pairs $k=4$, $5)$. Both modes rotate in the electron diamagnetic drift direction at a frequency of $\approx 6 \mathrm{kHz}$ and their maxima and minima are located at the sawtooth inversion radius, within experimental uncertainties. The toroidal mode number $n$, as obtained from the toroidal photodiodes, is such that $m=n$ for both modes. Although the modes appear to rotate in the poloidal direction, we believe that this rotation is primarily due to a nearly rigid toroidal rotation of the plasma and modes. 


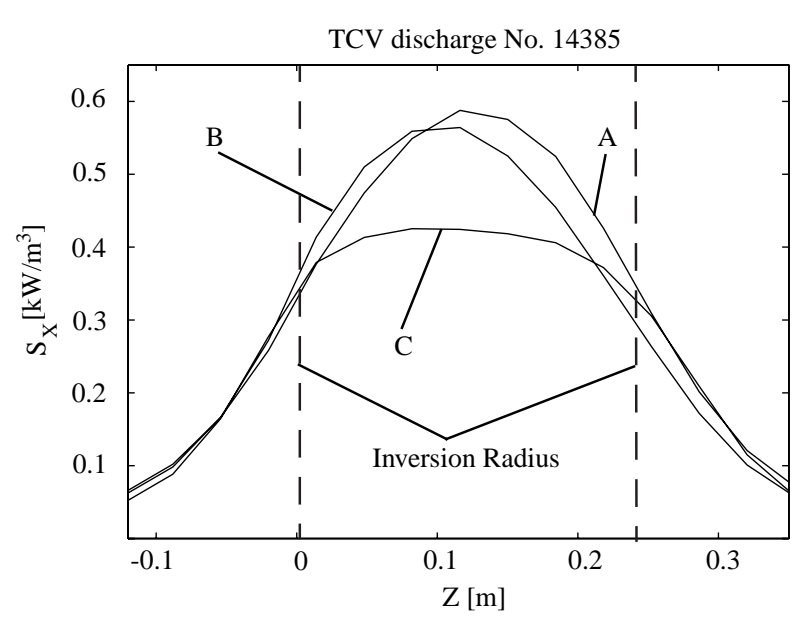

Figure 6. Soft X ray emissivity profiles along a central vertical cut corresponding to the different times indicated by the arrows in Fig. 4: A, the $S_{X}$ profile is poloidally symmetric at the end of the sawtooth ramp before the instability develops; B, during the sawtooth saturated phase the profile is not poloidally symmetric and the hot core is displaced by $\approx 1 \mathrm{~cm}$ from its position before the onset of the oscillations; $\mathrm{C}$, immediately after the sawtooth crash, $t-t_{\text {crash }} \approx 0.005 \tau_{\text {saw }}$, poloidal symmetry is restored. The dashed lines indicate the position of the sawtooth inversion radius.

The saturation phase terminates with the sawtooth crash when the rapid growth of the $m / n=1 / 1$ mode causes the hot core to move outwards in minor radius within $80 \mu \mathrm{s}$, see Fig. 4(d). Figure 6 shows different soft $\mathrm{X}$ ray emissivity profiles, at times corresponding to the arrows indicated in Fig. 4, together with the location of the sawtooth inversion radius. The poloidal symmetry is restored and the soft $\mathrm{X}$ ray emissivity profile is seen to flatten up to $r=r_{i n v}$ at the end of the sawtooth crash. The relative crash amplitude is less pronounced for a saturated sawtooth than for a standard sawtooth. In the present case $\Delta S_{X}(0) / \bar{S}_{X}(0) \approx 0.28$.

The lack of post-cursor oscillations on both soft $\mathrm{X}$ ray traces and Mirnov coil signals again suggests a full reconnection process. The absolute value of the displacement, $\xi_{\max } \approx 7 \mathrm{~cm}$, compared with the inversion radius, $r_{i n v} \approx 9.3 \mathrm{~cm}$, may be too small to explain a complete mixing of the plasma core inside $r_{m i x}$. However, it should be noted that in the very last phase of the reconnection process the maximum of the emissivity profile may no longer be at the position of the displaced magnetic axis in the hot core, making the determination of the displacement function $\xi$ difficult.

\subsection{Heating close to the sawtooth inversion surface}

In earlier ECH experiments on TCV [4], it was observed that small changes in the relative position between the ECH location and the sawtooth inversion surface can lead to large changes in the sawtooth shape, period and MHD activity. More recent experiments with three independent gyrotrons heating close to the sawtooth inversion surface suggested that even the beam width may play a role in determining the sawtooth shape [17]. In particular, two types of sawteeth are observed when the power is deposited close to the sawtooth inversion surface: large, almost triangular sawteeth, which we shall refer to as giant sawteeth, and non-standard sawteeth, which were termed humpback oscillations when they were observed in the T-10 tokamak [5]. A detailed study of the plasma parameters and heating conditions under which giant sawteeth occur, rather than humpbacks, has already been presented $[4,8,17]$. In what follows, an experimental account of the two types of sawteeth is given and an interpretation of the observed behaviour is proposed. Despite the strong difference in the soft $\mathrm{X}$ ray signals in the two types of sawteeth, we show that the gross features of the electron temperature behaviour and the MHD activity are similar and that they may result from the combined action of local ECH and a saturated magnetic island.

The sawtooth behaviour for giant sawteeth and the humpback oscillations is presented in Figs 7 and 8 , respectively. In both figures, the temporal evolution of the central soft $\mathrm{X}$ ray emissivity, $S_{X}(0)$, is shown in frame (a), together with the central electron density $n_{e}(0)$ from Abel inverted interferometer measurements, (b). The quantity $\tilde{T}_{e 0}=S_{X}(0) / n_{e}^{2}(0)$, in frame (c), is indicative of the evolution of the central electron temperature (the exponent $\gamma$ introduced above is close to unity in the present cases).

We examine the giant sawtooth case, for which the plasma parameters are $\delta=0.5, \kappa=1.47$, $I_{p}=330 \mathrm{kA}, q=3.25, P_{E C H} \approx 500 \mathrm{~kW}, \tau_{E}=$ $3.1 \mathrm{~ms}$ and $\tau_{\eta} \approx 300 \mathrm{~ms}$. The ECH absorption layer is located in the plasma midplane on the high field side of the magnetic axis inside the interval $r / a=[0.55,0.65]$. The sawtooth inversion surface is located at the normalized radius $r_{i n v} / a=0.6$. In Fig. 9, the electron temperature and density profiles are shown for the different times indicated by letters $\mathrm{A}-\mathrm{C}$ in Fig. 7. In this case, the Thomson scattering 


\section{Furno et al.}

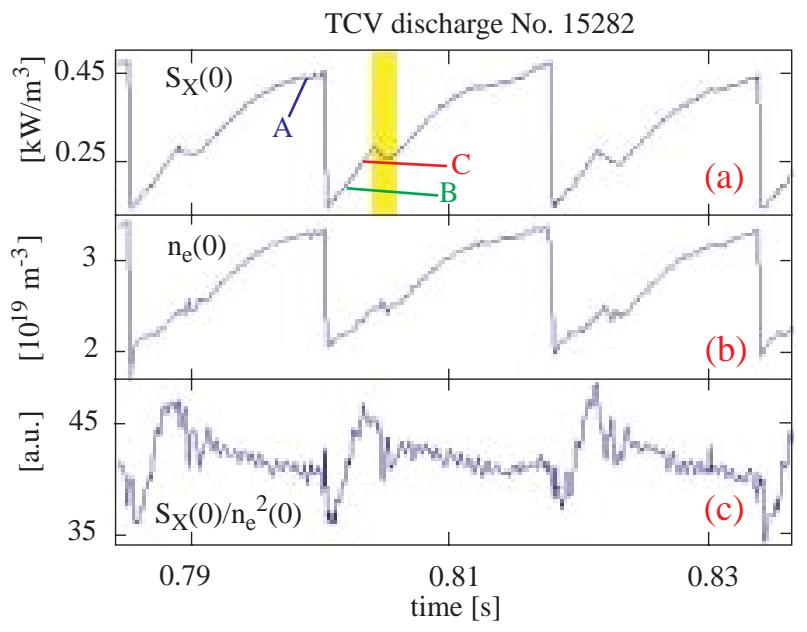

Figure 7. Giant sawteeth during TCV discharge 15282 with ECH power deposited close to the sawtooth inversion surface: (a) the central inverted soft $\mathrm{X}$ ray emissivity, $S_{X}(0)$; (b) the central electron density, $n_{e}(0) ;(\mathrm{c})$ a signal proportional to the variation of the electron temperature, $S_{X}(0) / n_{e}^{2}(0)$.

TCV discharge No. 16278

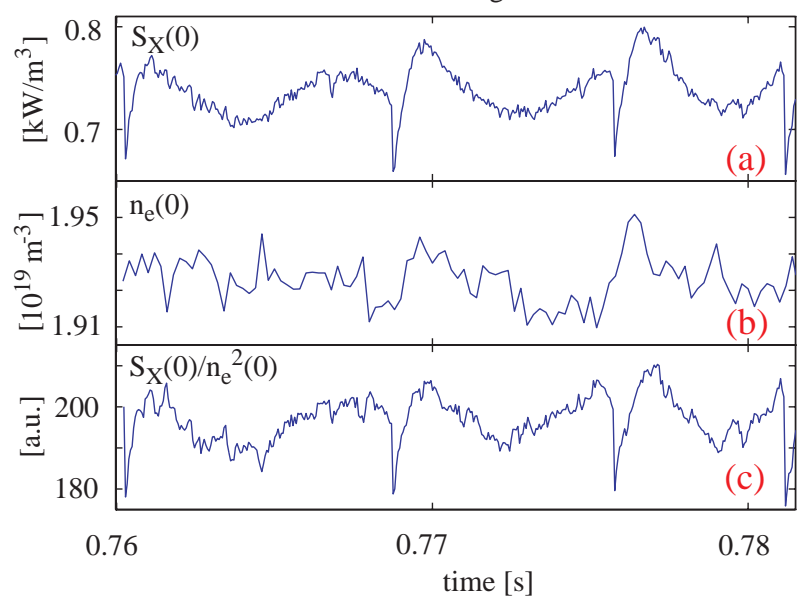

Figure 8. Humpback relaxation oscillations obtained with ECH power deposited close to the sawtooth inversion surface in TCV discharge 16278: (a) central inverted soft X ray emissivity, $S_{X}(0)$; (b) central electron density, $n_{e}(0)$; (c) a signal proportional to the variation of the electron temperature, $S_{X}(0) / n_{e}^{2}(0)$.

lasers were operated in the burst mode to obtain three profiles in a few milliseconds.

The sawtooth period, $\tau_{S T} \approx 15 \mathrm{~ms}$, is significantly longer than the standard sawtooth period. During a single sawtooth period, three distinct phases can be identified during which $\tilde{T}_{e 0}$ shows different
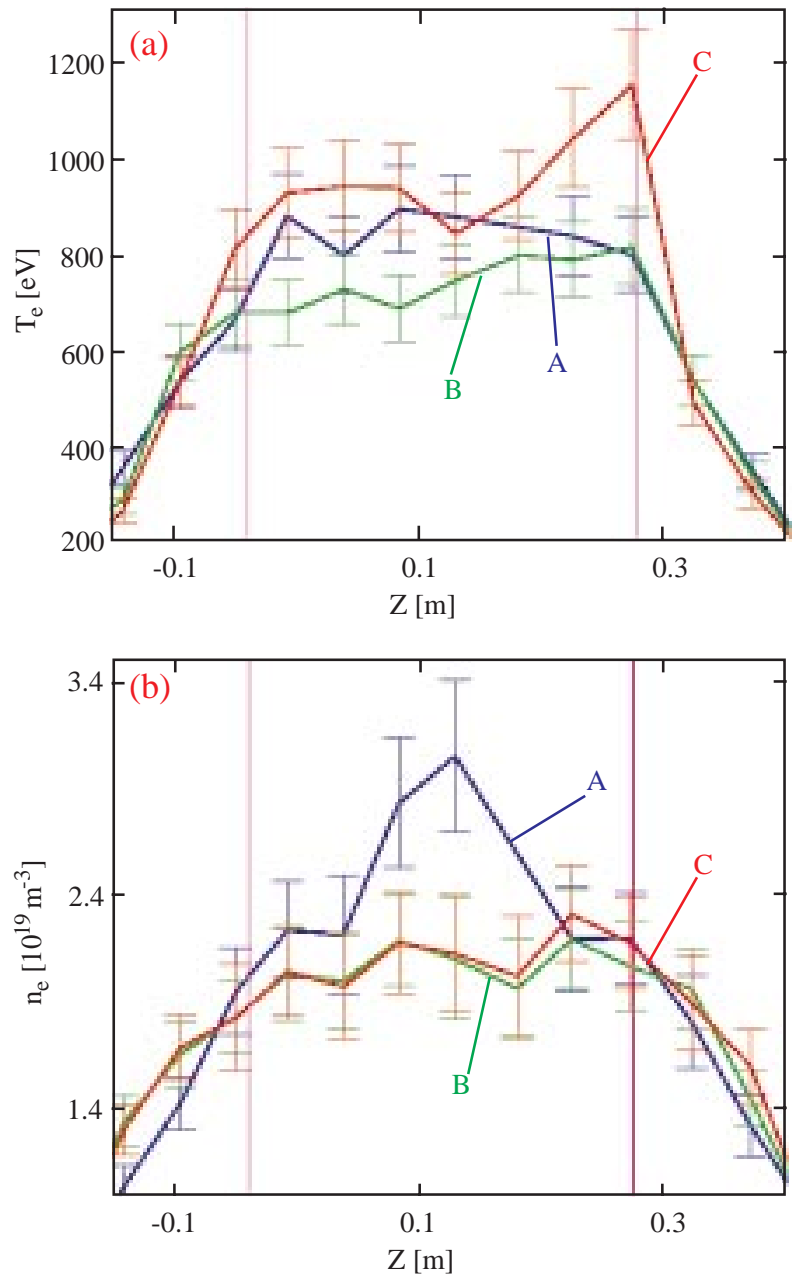

Figure 9. Thomson scattering profiles for TCV discharge 15282, shown in Fig. 7. The electron temperature (a) and the electron density (b) profiles relative to the times $t$ indicated by different colours in Fig. $7: t_{A}=$ $0.8004 \mathrm{~s}$, just before the sawtooth crash; $t_{B}=0.8014 \mathrm{~s}$, early during the sawtooth ramp; $t_{C}=0.8024$, before the onset of the oscillations. The position of the sawtooth inversion radius, from reconstructed soft X ray emissivity, is shown in magenta.

temporal behaviour (Fig. 7(c)). At the sawtooth crash, $\tilde{T}_{e 0}$ is observed to drop on a fast timescale, typically $\tau_{\text {crash }}<0.1 \mathrm{~ms}$. The pre-crash electron temperature profile (Fig. 9, profile A) is relatively flat inside the sawtooth inversion surface and the sawtooth crash results only in a small variation of the central electron temperature. Both the central soft $\mathrm{X}$ ray emissivity and electron density also drop during the crash phase. For instance, in Fig. 7, $\Delta S_{X}(0) / \bar{S}_{X}(0) \approx 0.66$ and $\Delta n_{e}(0) / \bar{n}_{e}(0) \approx 0.48$. The tomographic reconstructions of the soft $\mathrm{X}$ ray 
emissivity (not shown here) and the electron temperature profile (Fig. 9(b)) show that the poloidal symmetry is not restored after the sawtooth crash, suggesting an incomplete reconnection process.

The sawtooth crash is followed by a ramp phase during which the central electron temperature and density increase. The timescale of this phase, typically $\tau_{\text {ramp }} \approx 2 \mathrm{~ms}$, is slower than $\tau_{\text {crash }}$ and is the same as that of triangular sawteeth with central ECH deposition and comparable ECH power, safety factor and plasma energy content. The electron temperature profile C (Fig. 9) shows that the poloidal symmetry is not restored during the ramp phase, which terminates with the onset of strong oscillations in the soft $\mathrm{X}$ ray traces (highlighted in yellow in Fig. 7). Typical oscillations in the soft $\mathrm{X}$ ray signals due to the presence of a saturated magnetic island are not easy to distinguish early in the sawtooth ramp since the timescale of the rise is close to the rotation frequency of the mode which produces the oscillations (see below). In Fig. 10, the mode structure of the soft $\mathrm{X}$ ray emissivity during the oscillations is shown: a mode (Fig. 10, topos/chronos 2, 3) having poloidal mode number $m=1$ and rotating in the electron diamagnetic drift direction at a frequency of $\approx 1.5 \mathrm{kHz}$ is clearly present. The toroidal mode number is $n=1$. The sawtooth ramp is followed by a diffusive phase during which the central electron temperature undergoes small changes on an even slower timescale $\left(\tau_{\text {slow }} \approx 10 \mathrm{~ms}\right)$. During this phase, a strong influx of particles into the plasma core is seen, leading to a very peaked electron density profile. The profiles are poloidally symmetric and the electron temperature profile is flat inside the sawtooth inversion radius at the end of the sawtooth ramp. This phase lasts until the onset of precursor oscillations $\approx 500 \mu$ s before the subsequent sawtooth crash.

Figure 8 shows the humpback oscillation which can occur when heating close to the sawtooth inversion surface. The plasma parameters are $\delta=0.2$, $\kappa=1.37, I_{p}=200 \mathrm{kA}, q=4.8, P_{E C H} \approx 900 \mathrm{~kW}$, $\tau_{E}=3.6 \mathrm{~ms}$ and $\tau_{\eta} \approx 200 \mathrm{~ms}$. The ECH resonance position is located below the magnetic axis, resulting in a comparatively broader absorption layer located inside the interval $r / a=[0.2,0.4]$. The sawtooth inversion surface is located at the normalized radius $r_{\text {inv }} / a=0.32$.

For the humpback case, as for the giant sawteeth, $\tau_{S T}$ is significantly longer than the standard sawtooth period, $\tau_{S T} \approx 8 \mathrm{~ms}$. At first sight, the evolution of the central soft $\mathrm{X}$ ray emissivity in the
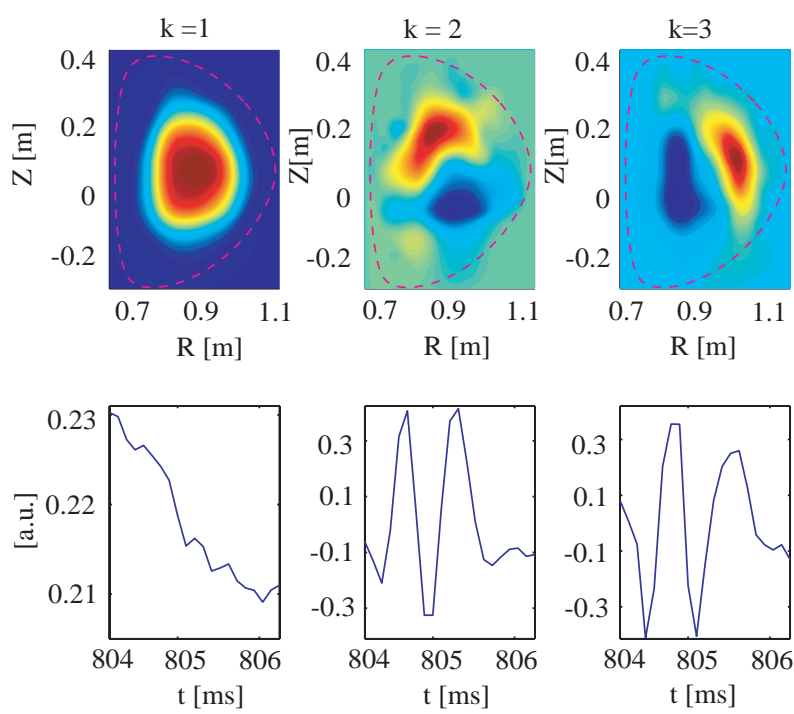

Figure 10. SVD analysis of the reconstructed soft X ray emissivity distribution during the phase of the giant sawtooth highlighted in yellow in Fig. 7. The top row shows the spatial eigenmodes (topos) with the LCFS (magenta dashed line). The corresponding temporal eigenvectors (chronos) are shown in the bottom row.

humpback case suggests that the electron temperature behaviour is radically different from that of the giant sawteeth. However, as can be seen from Fig. 8(c), the temporal evolution of the central soft $\mathrm{X}$ ray emissivity is mainly dependent on $\tilde{T}_{e 0}$, since $n_{e}(0)$ has only small variations (1-2\%) during a sawtooth period in the humpback case, as compared with the large variations of the density during the giant sawteeth. When this difference in the density behaviour is accounted for, the $\tilde{T}_{e 0}$ evolution is very similar. The three timescales of evolution of $\tilde{T}_{e 0}$, found in the giant sawtooth case, also apply to the humpback oscillations, and a detailed analysis of each of these phases, as previously performed for the giant sawteeth, reveals the same MHD mode structure.

The difference in the electron density behaviour between giant sawteeth and humpback oscillations is not yet understood, nevertheless we suppose that it can be attributed to the 'pump-out' effect observed in the presence of $m / n=1 / 1$ mode activity when sufficiently high ECH power is deposited inside the sawtooth inversion region in plasmas with modest triangularities [21]. In the case of the humpback oscillations, all these conditions are fulfilled. In particular, because of the broad ECH absorption region, a considerable amount of the power is deposited inside 


\section{Furno et al.}

the sawtooth inversion region resulting in a pumpout particle flux which prevents the electron density profile from peaking after the sawtooth crash. In the giant sawtooth case, the condition for the power deposited inside the inversion radius is not fulfilled because of the narrow ECH absorption region, and thus no pump-out effect is observed resulting in a peaking of the electron density during the sawtooth ramp which is comparable to that observed for triangular sawteeth with central heating.

Our interpretation for the electron temperature behaviour, in both sawtooth types described above, is based on the following heuristic picture. When the ECH power is deposited near the sawtooth inversion surface, the electron temperature profile tends to acquire distinctive features. In particular, $T_{e}$ becomes relatively flat (Fig. $9, T_{e}$ profile A), possibly even transiently hollow, in the central region up to $r=r_{i n v}$ and relatively steep outside this radius, as the result of localized heating and diffusive transport during a period of relative MHD quiescence. Since the formation of these features in the electron temperature profile requires a relatively long quiescent period, improved stability against $m / n=1 / 1$ modes is an important factor.

Different approaches to this stabilization can be envisaged through changes of the plasma pressure and/or current density profile. Recent simulations [22] using the PRETOR transport code [23], which contains a sawtooth trigger model based on a critical shear value at the $q=1$ surface $s_{1 \text { crit }}$ [24], suggest that the improvement in the $m / n=1 / 1$ mode stability, reported here, may result from a modification of the electron pressure gradient at the $q=1$ surface (resulting in an increased value of $\left.s_{1 \text { crit }}\right)$ and a slower growth of the magnetic shear at the $q=1$ surface after the sawtooth crash. The stabilization of the internal kink mode due to resonant interaction with suprathermal electrons [25] is not believed to be effective in the present case, since in TCV a suprathermal population of electrons is observed only with a significant toroidal injection angle [26]. This period of quiescence is terminated by the appearance of an $m / n=1 / 1$ magnetic island $[11,27]$, indicated by brief precursor oscillations in the soft X ray traces. In this first phase, as long as magnetic reconnection takes place, the island moves radially outwards and the soft $\mathrm{X}$ ray emissivity decreases. The magnetic topology during this phase, described in detail in Section 4, is such that crescent shaped surfaces inside the magnetic island separatrix directly connect the heated ring with the plasma centre. Therefore, the central plasma region is still heated even though radially separated from the $\mathrm{ECH}$ deposition region due to the large thermal conduction along the field lines. When the hot core starts to move outwards again and the reconnection process continues, the central temperature remains almost constant. The brief oscillations present in the experimental traces in this phase are in agreement with the existence of an $m / n=1$ mode and the picture of a magnetic island which is now close to the mixing radius, with the reconnection process close to completion. Subsequently, the poloidal symmetry is re-established and no further oscillations are seen in the soft $\mathrm{X}$ ray signals.

\section{Theoretical model}

Many of the experimental results in the previous section can be interpreted using a theoretical model, first presented in Ref. [10], which describes the evolution of the electron temperature in the presence of the $m / n=1$ magnetic island and localized heat sources. The derivation of this model is detailed in this section.

The part of the model that describes the evolution of the $m / n=1$ magnetic islands is fairly standard and the evolution of these islands is assumed to follow the characteristic spatial structure of a resistive internal kink mode [11] and the topological constraints of Kadomtsev's model [28]. Although this model is fairly old, its consequences in the presence of localized auxiliary heating have only recently been fully appreciated. In Ref. [10], it was proposed that the temperature 'filamentary' structures and sharp gradients observed in RTP [1] and in TEXT Upgrade [2] may be consistent with Kadomtsev's model. In this article, we show that the model may also account for the variety of the experimental observations in sawtoothing TCV discharges, although we do not strictly follow the Kadomtsev model.

We do not use Kadomtsev's prediction for the timescale of the sawtooth crash. This prediction would be valid for a plasma obeying resistive MHD equations [27]. High temperature tokamak experiments, such as TCV, are ill described by resistive MHD equations. For instance, TCV is better described by a semi-collisional kinetic model [4], where diamagnetic and ion Larmor effects are important for island evolution. Toroidal effects, not treated by Kadomtsev, are also important. Unfortunately, a fully consistent non-linear theory for the island dynamics that accounts for all these effects is not 
yet available. Therefore, the model used contains a free parameter, which describes the evolution of the $m / n=1$ magnetic island width, or alternatively, of the displacement function $\xi(t)$ which is measured experimentally, as shown in Section 3.

The original version of Kadomtsev's model assumes full magnetic reconnection. Single helicity $m / n=1$ island growth occurs until this island reaches its maximum amplitude, $w_{\max }=2 r_{\operatorname{mix}}$, where the mixing radius $r_{m i x}$ is defined in Section 4.1. We suspect that the single helicity assumption is not valid in a strict sense in a three dimensional toroidal system. Satellite harmonics of the internal kink magnetic perturbation localized within the $q=1$ volume, such as the $m / n=2 / 2$ harmonic driven by toroidal coupling, may cause some degree of magnetic stochasticity [29]. This, in turn, may cause the decoupling of the electron pressure and $q$ profiles: the pressure may flatten in the stochastic region independently of the $q$ profile. This would lead to incomplete reconnection and may explain why the safety factor on-axis $q_{0}$ is observed to remain below unity after the sawtooth crash in certain tokamak experiments $[30,31]$. According to this picture, the extent of the stochastic region determines the degree of departure from complete reconnection. For small departures, the model presented here may satisfactorily reproduce the gross features of the experimental observations. Conversely, we are tempted to conclude that if the model results agree with the experimental data, then full reconnection is really obtained experimentally. A direct measurement of the $q$ profile would settle this issue, but unfortunately such a measurement is not available on TCV.

\subsection{Derivation of the model}

We assume, for simplicity, a cylindrical plasma column of length $L$ with periodic boundary conditions. In the absence of an $m / n=1$ island, the magnetic flux surface cross-sections are concentric circles of constant normalized helical flux,

$\Psi_{*_{\text {in }}}(r)=\int_{0}^{r^{2}}\left[q_{\text {in }}(x)^{-1}-1\right] d x$

where $x=r^{2}$ and the subscript 'in' stands for initial, i.e. before island formation. An elliptical deformation of the flux surfaces with constant elongation can be introduced trivially if desired. For a typical case (i.e. a monotonic $q$ profile), $\Psi_{*_{i n}}(r)$ has a maximum at the $q=1$ radius, $r=r_{s}$, as shown in Fig. 11(a).

Our problem is now to specify the helical flux function $\Psi_{* i n}(r, \alpha, t)$ during the growth of the

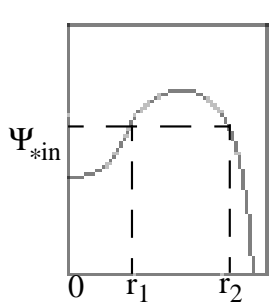

(a)

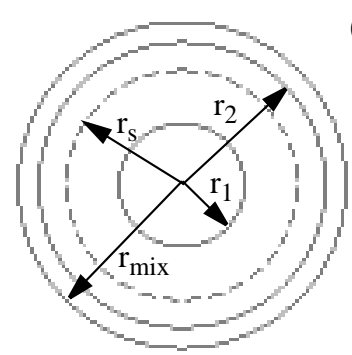

(b)
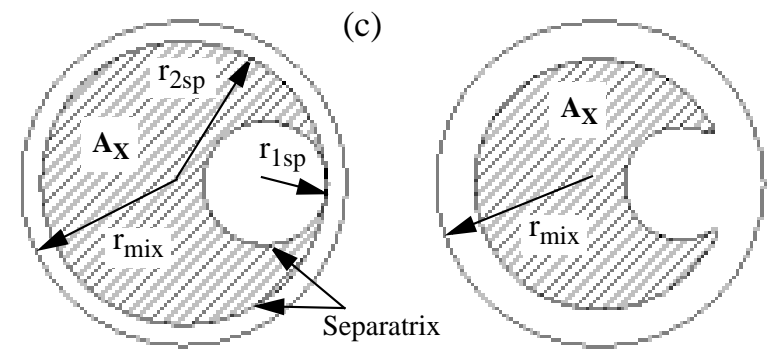

(d)

Figure 11. Magnetic reconnection process according to the Kadomtsev model: (a) pre-crash helical flux for a monotonic $q$ profile; (b) helical flux surfaces before reconnection starts; (c) the flux surface originally at $r=r_{1}$ reconnects with the flux surface, corresponding to the same helical flux, originally at $r=r_{2}$; (d) at a later time, the separatrix of (c) has deformed into a crescent shaped surface maintaining a constant area $A_{X}$.

$m / n=1$ island, where $\alpha=\vartheta-2 \pi z / L, \vartheta$ being the poloidal angle. We note that this function is not explicitly determined in Kadomtsev's paper [28], where only $\Psi_{*_{r e l}}(r)$ is derived as a function of $\Psi_{* i n}(r)$, with the subscript 'rel' referring to the relaxed helical flux at the full reconnection. Nevertheless, the two basic rules for the reconstruction of $\Psi_{* i n}(r, \alpha, t)$ are to be found in that paper. These rules are:

(a) Helical flux conservation, i.e.

$$
\frac{\partial}{\partial t} \Psi_{*}+\boldsymbol{v} \cdot \nabla \Psi_{*}=0
$$

which describes the evolution of $\Psi_{*}$ everywhere except in a narrow layer around the island $\mathrm{X}$ point where magnetic reconnection occurs,

(b) Toroidal flux conservation.

These rules do not define a unique solution for the functional $\Psi_{*_{i n}}(r, \alpha, t)$, although they represent a strong mathematical constraint. A prescribed model for the velocity field $\boldsymbol{v}$ is assumed corresponding to a resistive internal kink motion. Accordingly, helical flux surfaces within the $q=1$ surface are shifted rigidly. The motion is thus specified by a single 


\section{Furno et al.}

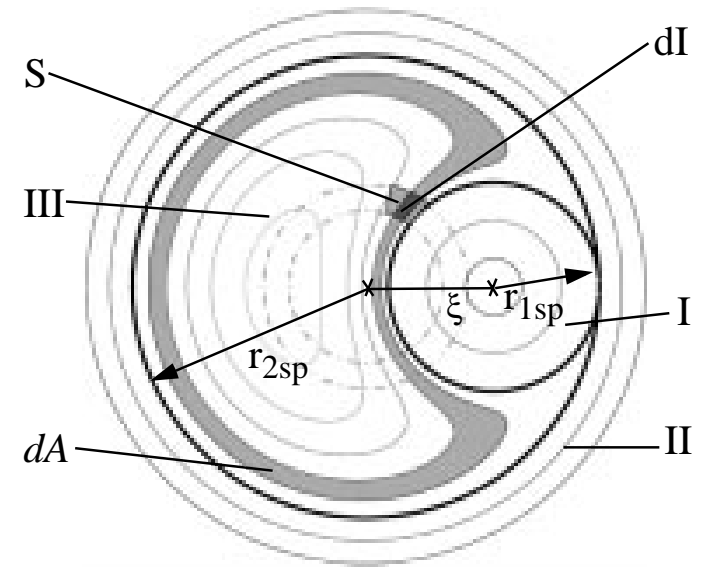

Figure 12. Magnetic topology during reconnection. The island separatrix is formed by the circles of radii $r_{1 s p}$ and $r_{2 s p}$, and $\mathrm{S}$ is the region where the $\mathrm{ECH}$ power is deposited. Outside the island separatrix (regions I and II) the magnetic flux surfaces are circular, while inside (region III) they are crescent shaped. In the plasma rest frame, with the assumption of rigid toroidal rotation, the toroidally averaged heated region is the ring indicated by chain circles. $d I$ shows the intersection of the heated ring with a generic flux tube of cross-sectional area $d A$.

function $\xi(t)$, representing the radial displacement of the magnetic axis from its original position. The assumption of a given velocity field greatly simplifies the analysis, but is not fully self-consistent with the solution of the set of resistive MHD equations. An improved analytical model was the one proposed by Waelbroeck [32]. Alternatively, the resistive MHD equations could be solved numerically, which is not, however, in the spirit of the present analysis.

According to the procedure outlined above, at each time $t$ during the reconnection process, the island separatrix is formed by two circles of radii $r_{1 s p}<r_{s}$ and $r_{2 s p}>r_{s}$, where $\Psi_{*_{i n}}\left(r_{1 s p}\right)=$ $\Psi_{* i n}\left(r_{2 s p}\right)$, with the centre of the inner circle (i.e. the original magnetic axis) displaced from its original position by a distance

$\xi(t)=r_{2 s p}-r_{1 s p}$

(Fig. 12). With $B_{z}=$ const, toroidal flux conservation is equivalent to area conservation. Thus, the area $A_{X}$ enclosed by the magnetic separatrix equals that of the annular region between the initial position of the two reconnecting circles, i.e. $A_{X}=\pi\left(r_{2 s p}^{2}-r_{1 s p}^{2}\right)$.

The magnetic island has a width

$w_{i s l}(t)=2 \xi(t)$.
At a later time, the island separatrix evolves into a crescent shaped surface, maintaining a constant area $A_{X}$ indicated by the dashed regions in Fig. 11(d). The contour of the crescent is also a surface of constant helical flux, specifically

$\Psi_{* i n}(r, \alpha, \xi)=\Psi_{*_{i n}}\left(r_{1 s p}\right)$

where the function $\Psi_{*}$ now depends on time through $\xi(t)$. Eventually, at full reconnection when poloidal symmetry is restored, each reconnected surface becomes a circle of radius $r_{k}$ such that $\pi r_{k}^{2}=A_{X}$, or

$r_{k}^{2}=r_{2 s p}^{2}-r_{1 s p}^{2}$.

The relaxed helical flux must satisfy the relationships

$\Psi_{*_{r e l}}\left(r_{k}\right)=\lim _{\xi \rightarrow r_{\text {mix }}} \Psi_{\left.*\right|_{r=r_{k}}}=\Psi_{*_{i n}}\left(r_{1 s p}\right)$.

The magnetic axis of the initial configuration reconnects with the flux surface at $r=r_{m i x}$, such that

$\Psi_{*_{i n}}(0)=\Psi_{*_{i n}}\left(r_{m i x}\right)$.

This formula defines the mixing radius. Observe that, at full reconnection, the initial magnetic axis and the island $\mathrm{O}$ point annihilate each other, while the new axis of the relaxed configuration corresponds to the O point of the expanding $m / n=1$ island.

So far, we have specified the constraints that the function $\Psi_{*}$ must obey but we have not derived this function explicitly. This is now done in two steps. In the first step, we find it convenient to introduce a Hamiltonian function, $H=H\left(\Psi_{*}\right)=H(r, \alpha, \xi)$, whose constant energy contours correspond to the crescent shaped surfaces within the island separatrix. This region is indicated as region III in Fig. 12. A suitable choice for this function, details of which are given in Ref. [10], is

$H=\frac{\left(r^{2}+\xi^{2}-r_{1 s p}^{2}-2 r \xi \cos \alpha\right)\left(r_{2 s p}^{2}-r^{2}\right)}{r^{2}+\xi^{2}-2 r \xi \cos \alpha}$.

The parameters $r_{1 s p}$ and $r_{2 s p}$ can readily be obtained as a function of $\xi(t)$ and $\Psi_{* i n}(r)$, which are both assumed to be given. The island separatrix for a given $\xi$ corresponds to the contour level $H=0$, while $H$ reaches a maximum value $H_{\max }(\xi)$ on the island O point. Crescent shaped surfaces correspond to contour levels $H=H_{0} \in\left[0, H_{\max }\right]$. Note that the curves $H(\alpha, r, t)=H_{0}$ become circles in both the limits $\xi=0$ and $\xi=r_{m i x}$ (in the latter limit, $r_{1 s p}=0$ ).

In the second step, the function $A=A(H, \xi)$ can be evaluated numerically after computing the area 
$A_{X}$ pertaining to each $H=H_{0}$ surface; from this, using the area conservation rule, the function $\Psi_{*}=$ $\Psi_{*}(H)$ can be constructed. A simple analytic fit can also be obtained,

$A(H, \xi)=A_{X}(\xi)\left(1-\frac{H}{H_{0}}\right)$.

For an inverse parabolic $q$ profile,

$\frac{q_{0}}{q_{\text {in }}\left(r^{2}\right)}=1-\Delta q\left(\frac{r}{r_{s}}\right)^{2}$

where $\Delta q=1-q_{0}$ is positive definite, we obtain [28]

$\Psi_{* r e l}\left(r^{2}\right)=\frac{\Delta q}{2 q_{0}} r_{s}^{2}\left(1-\frac{r^{4}}{4 r_{s}^{4}}\right)$

and through Eqs (6)-(8) and (11),

$\Psi_{*}(\alpha, r, \xi)=\frac{\Delta q}{2 q_{0}} r_{s}^{2}\left[1-\frac{A_{X}^{2}(\xi)}{4 \pi^{2} r_{s}^{4}}\left(1-\frac{H(\alpha, r, \xi)}{H_{0}(\xi)}\right)^{2}\right]$.

This function $\Psi_{*}$ is valid within the island region III of Fig. 12. In regions I and II, the magnetic surface cross-sections and the function $\Psi_{*}$ are trivially determined from $\Psi_{* i n}(r)$.

Let us now derive an equation for the electron temperature $T_{e}$. Since in a magnetized high temperature plasma the heat transport along magnetic field lines is large compared with the transport perpendicular to the magnetic field lines, the electron temperature is constant on magnetic flux surfaces, i.e. $T_{e}=T_{e}\left(\Psi_{*}, t\right)$. Similarly, since electron parallel diffusion is very large, we may conclude that $n_{e}=n_{e}\left(\Psi_{*}, t\right)$. Surfaces of constant $\Psi_{*}$ move with the fluid and it is convenient to express the heat transport equation in a Lagrangian frame. We consider the limit of intense electron heating and relatively low density, where the collisional energy transfer between electrons and ions can be neglected. This is certainly the case in TCV with intense $\mathrm{ECH}$, where the electron temperature can exceed the ion temperature by a factor of 10 . The relevant equation becomes

$\frac{3}{2} n_{e} \frac{\partial T_{e}}{\partial t}=-\nabla \cdot \boldsymbol{q}+S$

where $S$ is the heat source and

$\boldsymbol{q}=-n_{e} \chi_{\perp} \nabla_{\perp} T_{e}$

is the heat flux, with $\chi_{\perp}$ the perpendicular heat diffusion coefficient. Flux surface averaging (denoted by angular brackets) of Eq. (15) gives

$\frac{3}{2} n_{e} \frac{\partial T_{e}}{\partial t}=\left\langle\nabla \cdot\left(n_{e} \chi_{\perp} \frac{\partial T_{e}}{\partial \Psi_{*}} \nabla \Psi_{*}\right)\right\rangle+\langle S\rangle$.
This equation is coupled to the equation for the electron density, which reads

$$
\frac{\partial n_{e}}{\partial t}=\left\langle\boldsymbol{\nabla} \cdot\left[\left(D_{\perp} \frac{\partial n_{e}}{\partial \Psi_{*}}-\frac{n_{e} v_{P e}}{\left|\nabla \Psi_{*}\right|}\right) \nabla \Psi_{*}\right]\right\rangle
$$

where $D_{\perp}$ is the electron diffusion coefficient across the field lines and $v_{P e}$ is the pinch velocity. Equations (17) and (18) describe the evolution of the electron density and temperature on magnetic flux tubes frozen to the plasma flow. At a particular instant in time when two flux tubes with equal helical fluxes reconnect, mixing rules for the particle density and thermal energy density must be implemented, which means that both the temperature and the density evolve during the reconnection process even if $\chi_{\perp}=S=0$.

The mixing rules are derived from the particle and energy conservation relations. Particle conservation can be written in integral form as

$\int_{A} n_{e}\left(A^{\prime}\right) d A^{\prime}=\pi \int_{r_{1}^{2}}^{r_{2}^{2}} n_{\text {in }}\left(r^{2}\right) d r^{2}$

where, as before, $A$ is the area between two circles with equal helical fluxes before they reconnect. After the reconnection, $A$ is the area of the crescent shaped surface with the same value of $\Psi_{*}$. Differentiating Eq. (19), we obtain

$n_{e}\left[A\left(\Psi_{*}\right)\right]=\pi\left(n_{e}\left(r_{2 s p}^{2}\right) \frac{d r_{2 s p}^{2}}{d A}-n_{e}\left(r_{1 s p}^{2}\right) \frac{d r_{1 s p}^{2}}{d A}\right)$

where the metric elements $d r_{1 s p}^{2} / d A$ and $d r_{2 s p}^{2} / d A$ can be obtained from the initial $\Psi_{*_{i n}}(r)$. For instance, for the inverse parabolic $q$ profile in Eq. (12), one finds

$-d r_{1 s p}^{2} / d A=d r_{2 s p}^{2} / d A=1 /(2 \pi)$.

The mixing rule for the thermal energy is obtained from similar considerations. However, we should consider that magnetic energy is transformed into heat during the reconnection process. A simple calculation shows that typically the transformed magnetic energy is smaller than the energy associated with the equilibrium poloidal magnetic field by two orders of magnitude and therefore can be neglected. Thermal energy conservation gives

$$
\begin{aligned}
p_{e}\left[A\left(\Psi_{*}\right)\right] & =\pi\left(p_{e}\left(r_{2 s p}^{2}\right) \frac{d r_{2 s p}^{2}}{d A}-p_{e}\left(r_{1 s p}^{2}\right) \frac{d r_{1 s p}^{2}}{d A}\right) \\
& =n_{e}\left(\Psi_{*}\right) T_{e}\left(\Psi_{*}\right) .
\end{aligned}
$$

Since $p_{e}=n_{e} T_{e}$, Eqs (20) and (22) can be combined to give the mixing rule for the electron temperature. 


\section{Furno et al.}

The appropriate boundary conditions for the temperature are as follows: an initial temperature $T_{e}(r, t=0)=\tilde{T}(r)$, where $\xi(t=0)=0$; a condition at the plasma edge, $r=a$, located in region II of Fig. 12, normally $T(a, t)=0$; the geometrical conditions $\partial T_{e} /\left.\partial \tilde{r}\right|_{I, I I}=0$, where $\tilde{r}$ is the distance from the magnetic axis in the two regions. Finally, a condition at the separatrix is required. Note that, with $\chi_{\perp}=0$, a discontinuity of $n_{e}$ and $T_{e}$ across the separatrix is in general allowed by Eq. (22). With finite $\chi_{\perp}$, a common temperature is found, approaching the separatrix from any of the three regions I, II and III. This common temperature is determined by the continuity of the heat flux. The boundary conditions for the density are similar. Equations (17), (18), (20), (22) with the appropriate boundary conditions and the determination of the helical flux function, as described above, completely specify the theoretical model.

In applying our model to the interpretation of the TCV experimental results, a number of simplifying assumptions have been made:

(a) Rigid plasma toroidal rotation with a period $\tau_{\text {rot }}$.

(b) The ECH power source $S$ is located on the poloidal midplane between radii, $r_{h 1}, r_{h 2}$. The radial width is dominated by the finite height of the wave beam. As a result of the observed fast plasma rotation and the presence of the $m / n=1 / 1$ island, the deposition is effectively spread around the poloidal cross-section in the shape of a ring indicated by the chain circles in Fig. 12. This average approach is justified as long as the changes in the flux surface geometry are slow compared with the plasma rotation velocity, i.e. when

$\tau_{\text {rot }} \ll \frac{1}{\xi} \frac{d \xi}{d t}$.

For instance, this condition does not apply during the sawtooth crash phase. The region obtained by rotating the heated ring in the toroidal direction has a volume $V_{h}$. Thus, in the plasma frame, we take for simplicity

$S=\rho \mathcal{H}\left[\left(r-r_{h 1}\right)\left(r_{h 2}-r\right)\right]$

where $\mathcal{H}$ is the Heaviside function and $\rho=P_{E C H} / V_{h}$. Since parallel heat conduction is dominant, the deposited heat spreads rapidly over all flux tubes intersecting the heated ring. Thus, if we denote by $d A$ the cross-sectional area of a generic flux tube and by $d I$ its intersection with the heated ring, we can write the ECH power density averaged over flux surfaces as $\langle S\rangle(A)=\rho d I / d A$. Note that the heat is transported radially by parallel diffusion in a complex magnetic structure, such as that of Fig. 12, resulting in an apparent non-local transport process.

(c) The perpendicular heat diffusion coefficient $\chi_{\perp}$ is taken to be of the form

$\chi_{\perp}=\left(\frac{r}{a}\right)^{\beta} \chi_{\max }$

for $r \geq r_{2 s p}$ and

$\chi_{\perp}=\left(\frac{r_{2 s p}}{a}\right)^{\beta} \chi_{\max }=$ const

for $r<r_{2 s p}$ with $\chi_{\max }$ and $\beta$ constant parameters; $r \geq r_{2 s p}$ corresponds to region II of Fig. 12, where $\Psi_{*}=\Psi_{*}{ }_{\text {in }}(r)$.

(d) The initial $q$ profile is taken as parabolic, Eq. (12). Note that this profile is used only up to $r=r_{m i x}$; for this profile, $r_{\text {mix }}=\sqrt{2} r_{s}$. For $r \geq r_{\text {mix }}$, the magnetic topology is unchanged and the $q$ profile can differ from inverse parabolic. The precise value of $q_{0}$ is found not to affect the resulting temperature evolution. Electron cyclotron current drive effects are neglected.

(e) The electron density profile is flat inside the mixing radius and fixed during the sawtooth period. This is the crudest of all our simplifications. It is justified on the basis that, with intense $\mathrm{ECH}$, the electron temperature profile tends to become rather peaked and, during a sawtooth period, $\Delta T_{e} / T_{e} \gg$ $\Delta n_{e} / n_{e}$. This condition is satisfied for the standard and saturated sawteeth discussed in Section 3, but is only marginally satisfied for humpback oscillations and giant sawteeth.

(f) The temperature profile at $t=0$ is chosen to be

$T(r, t=0)=T_{0}\left(1-\frac{r^{2}}{a^{2}}\right)$

with $T_{0}=1 \mathrm{keV}$. However, after an initial transient, which may last for a few sawtooth periods, the temperature evolution settles into a periodic cycle independent of $T(r, t=0)$.

\section{Simulation results}

The numerical simulations are discussed in this section. The parameters used in the simulations are given in Table 1. 
Table 1. Simulation parameters

\begin{tabular}{lccccc}
\hline Simulation & $r_{h 1} / r_{\operatorname{mix}}$ & $r_{h 2} / r_{\operatorname{mix}}$ & $\chi_{\max }\left(\mathrm{m}^{2} / \mathrm{s}\right)$ & $\beta$ & $P_{E C H}(\mathrm{~kW})$ \\
\hline Standard sawteeth & 0 & 0.25 & 3.5 & 1.8 & 500 \\
Saturated sawteeth & 0 & 0.25 & 10.5 & 3 & 460 \\
Deposition on $q=1$ & 0.55 & 0.8 & 4.8 & 2 & 450 \\
\hline
\end{tabular}

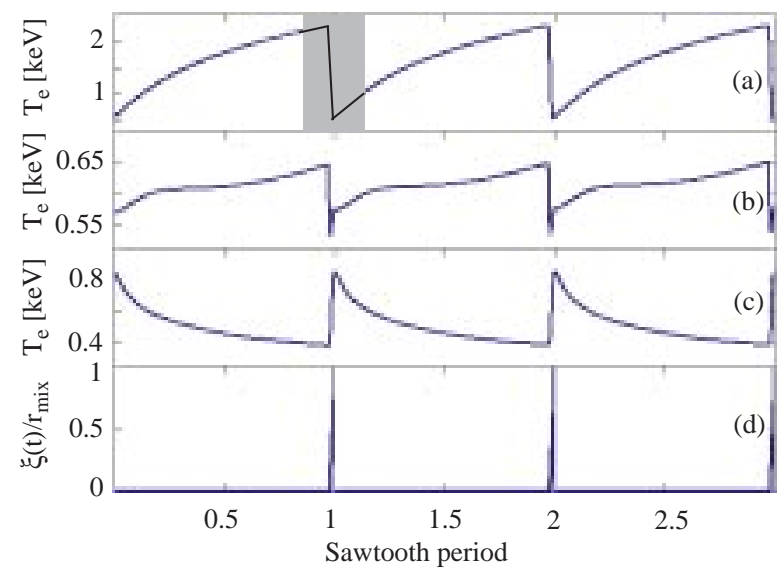

Figure 13. Simulation of triangular sawteeth with onaxis ECH power deposition. The electron temperature is shown at different radial positions: (a) on-axis, $r=0$; (b) just inside the inversion radius, $r=0.85 r_{\text {inv }}$; (c) just outside the inversion radius, $r=1.27 r_{i n v}$; (d) displacement function $\xi(t) / r_{\operatorname{mix}}$.

\subsection{Standard sawteeth}

Figure 13 shows the simulation results of standard triangular sawteeth in the presence of central ECH. The function $\xi(t)$ is taken to be zero for $0 \leq t \leq 0.975 \tau_{\text {saw }}$ and then to increase linearly up to $r_{\text {mix }}$ during the crash, i.e. for $0.975 \tau_{\text {saw }} \leq t \leq \tau_{\text {saw }}$, consistent with the experimental displacement function shown in Fig. 1. Figure 13 shows local temperature traces and Fig. 14 the simulated temperature profiles corresponding to different times in the shaded box of Fig. 13. The most striking experimental feature reproduced by our simulation is the formation of a hot ring in the relaxed temperature profile. This is not observed in TCV ohmic discharges, but it occurs with intense central ECH when the pre-crash temperature profile is very peaked. The formation of the hot ring is a straightforward consequence of Kadomtsev's full reconnection model for cases where the crash phase is very rapid, so that diffusion and heating can be neglected during this phase, and the pre-crash temperature profile is

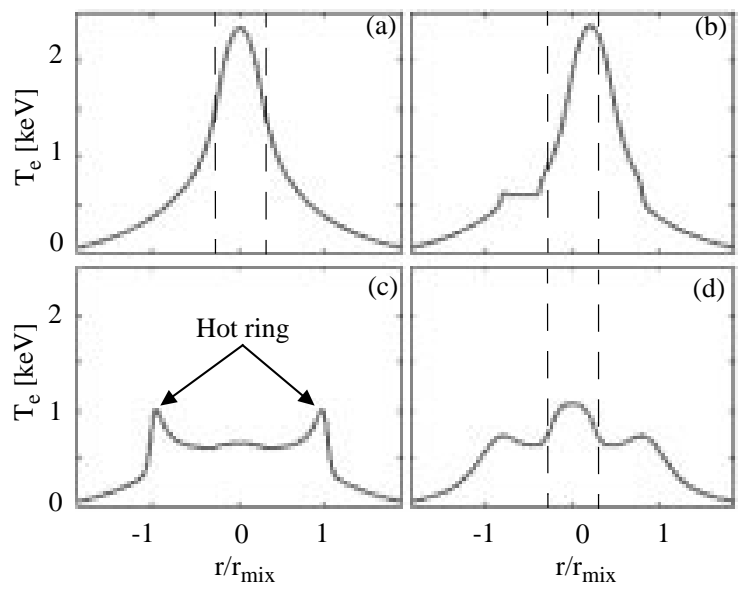

Figure 14. Simulated electron temperature profiles for the time window indicated in Fig. 13: (a) pre-crash, $\xi=$ 0 ; (b) during hot core displacement, $\xi=0.41 r_{\text {mix }}$; (c) just after the sawtooth crash, $\xi=r_{m i x}$, the hot ring is formed; (d) early during reheat ramp, corresponding to $t-t_{\text {crash }}=$ $0.125 \tau_{\text {saw }}$. The power deposition region is indicated by the dashed lines.

sufficiently peaked. An analytic calculation provides a better understanding of this point. Assume, for simplicity, a constant density profile, a pre-crash $q$ profile as in Eq. (12) and a rather peaked pre-crash temperature profile,

$T_{i n}(r)=T_{0}\left(1-\frac{r^{2}}{a^{2}}\right)^{\alpha}$

with $\alpha=2$.

Using Eq. (22), the relaxed temperature profile is

$T_{r e l}(r)=T_{i n}\left(r_{s}\right)\left(1+\frac{r^{4}}{4\left(a^{2}-r_{s}^{2}\right)^{2}}\right)$

for $r \leq r_{\text {mix }}$, while for $r>r_{m i x}, T_{r e l}(r)=T_{i n}(r)$. This profile is hollow up to $r=r_{\text {mix }}$ and the discontinuity at $r=r_{\operatorname{mix}}$ would be smoothed out by perpendicular heat diffusion. The resulting profile has a hot ring structure centred in the proximity of $r_{m i x}$.

In general, a hot ring forms if $\alpha>1$ in Eq. (26). For less peaked temperature profiles with $\alpha \leq 1$, as 


\section{Furno et al.}

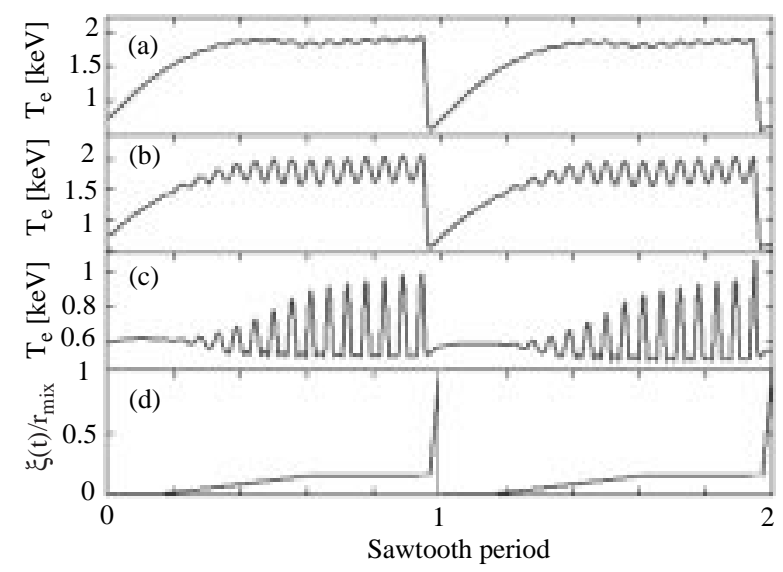

Figure 15. Simulation of saturated sawteeth with onaxis ECH power deposition. The electron temperature is shown at different radial positions: (a) close to the magnetic axis, $r=0.01 r_{i n v}$; (b) slightly off-axis, $r=0.15 r_{i n v}$; (c) just inside the inversion radius, $r=0.85 r_{i n v}$, where frequency doubling is visible; (d) the hot core radial displacement $\xi(t) / r_{\text {mix }}$.

more typical for ohmic discharges, the relaxed profiles is flat or perhaps slightly peaked on-axis. For instance, assuming $\alpha=1$, a constant density profile and an inverse parabolic $q$ profile as in Eq. (12), Eq. (22) yields a constant temperature up to $r=r_{\text {mix }}, T_{\text {rel }}=T_{0}=T_{\text {in }}\left(r_{1}\right)$.

\subsection{Saturated sawteeth}

Saturated sawteeth can be obtained with our model with central or slightly off-axis heating, provided the heat is deposited well within the $q=1$ radius, Fig. 15, with the simulation parameters in Table 1. The key to obtaining saturated sawteeth is to assume a displacement function which moves the magnetic axis early in the sawtooth ramp, by a distance comparable to the outer radius of the heat deposition region. In this way, the heating power is no longer deposited near the magnetic axis. Consequently, the electron temperature saturates at the plasma centre, i.e. at the original position of the magnetic axis before the onset of the $m / n=1$ island. The displacement function used for this simulation, shown in Fig. 15(d), was chosen to reproduce the experimentally measured displacement, shown in Fig. 4.

With a magnetic island width, $w_{i s l}=2 \xi$, the temperature profile is not poloidally symmetric. In the presence of toroidal plasma rotation, this gives rise to oscillations of the simulated electron temperature

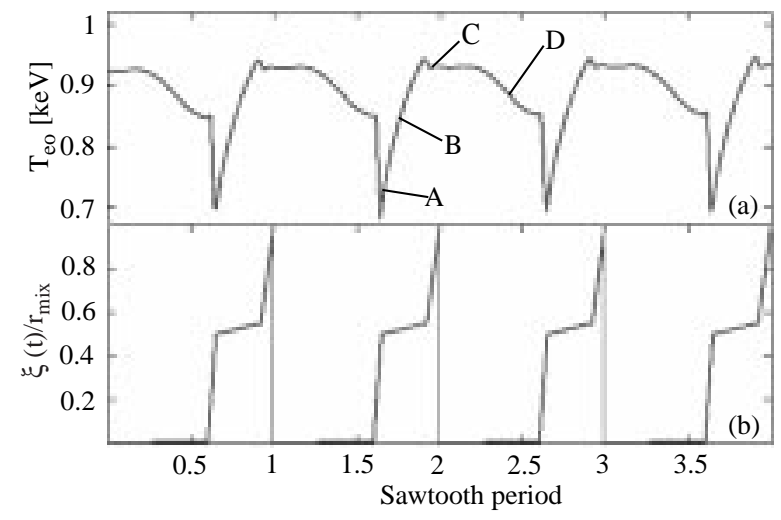

Figure 16. Simulation results of sawtooth oscillations with ECH power deposited close to the $q=1$ surface: (a) the central electron temperature, (b) the displacement function $\xi(t) / r_{\text {mix }}$.

traces, Fig. 15(b, c). Of particular interest is the doubling of the oscillation frequency, in good agreement with the experimental traces, Fig. 4.

\subsection{Deposition close to the sawtooth inversion surface}

Recent attempts to simulate the humpback oscillations [33], using the same model, resulted in a sawtooth shape in which the drop and rise of the central electron temperature occur on the same timescale. This was mainly due to the choice of the displacement function $\xi(t)$ and to the assumption of full magnetic reconnection after the sawtooth crash. This condition was, however, not fulfilled in the two examples presented in Section 3. In the present simulation, the function $\xi(t)$ shown in Fig. 16(b) increases rapidly up to $0.5 r_{\text {mix }}$ during the crash and then remains constant during the sawtooth ramp, i.e. for $0 \leq t \leq 0.3 \tau_{\text {saw }}$. After the sawtooth ramp, for $0.3 \tau_{\text {saw }} \leq t \leq 0.4 \tau_{\text {saw }}, \xi(t)$ reaches the mixing radius, completing the reconnection process. The ECH power is deposited on the $q=1$ surface and the parameters are given in Table 1 . The main features of experimental sawtooth traces are reproduced. In particular, the overall evolution of the central electron temperature is in agreement with the time traces shown in Figs 7 and 8 . The three distinct phases with different timescales, described previously, are correctly taken into account by the simulation and the electron temperature profiles are qualitatively consistent with the Thomson scattering measurements, Fig. 9. 


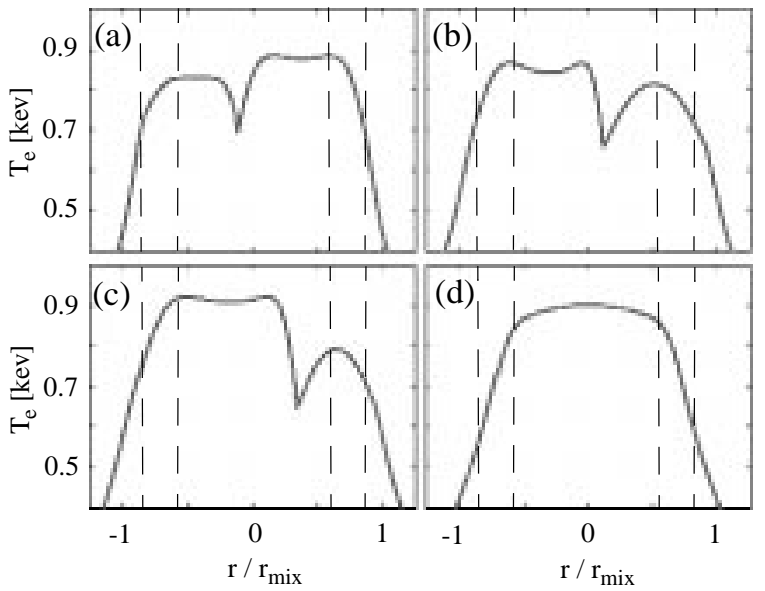

Figure 17. Simulated electron temperature profiles obtained with ECH power deposited on the $q=1$ surface. The $T_{e}$ profiles shown in (a), (b), (c) and (d) correspond, respectively, to the times $\mathrm{A}, \mathrm{B}, \mathrm{C}$ and $\mathrm{D}$ indicated in Fig. 16: (a, b) post-crash profiles during sawtooth ramp, $\xi=0.5 r_{\text {mix }}, \xi=0.518 r_{\text {mix }}$; the profiles are not poloidally symmetric since the reconnection process is incomplete; (c) during hot core displacement, $\xi=0.719 r_{\text {mix }}$; (d) at complete magnetic reconnection, $\xi=r_{m i x}$. The power deposition region is indicated by the dashed lines.

\section{Conclusions}

In the TCV tokamak, different types of sawtooth oscillations, ranging from triangular to non-standard sawteeth, are observed in the presence of ECH as the location of the power deposition is moved from the magnetic axis to the sawtooth inversion surface. Measurements with high temporal resolution have shown that the different sawtooth shapes and their features in the electron temperature profiles are compatible with the strong localization of the heating region, which is specific to the ECH method, and of the advection and mixing of electron thermal energy associated with a resistive internal kink mode. This mode produces precursor oscillations which have been identified to have $n=1$ and dominant $m=1$ mode numbers for all sawtooth types. This shows that all the different sawtooth relaxation cycles can be reproduced by a single model. The detailed dynamics of the magnetic island, associated with the resistive internal kink mode, is described by the displacement function $\xi(t)$ [11], which is inferred from the experimental data for the different sawtooth types.

In triangular sawteeth, obtained with on-axis heating, the $m / n=1 / 1$ mode develops immediately before the crash, whereas, when the deposition is offaxis but still within the sawtooth inversion surface, it grows early in the sawtooth ramp and remains at a saturated level until the sawtooth crash, resulting in saturated sawteeth. In both cases, the sawtooth crash is consistent with a full reconnection process and its overall effect is to expel particles and heat from the plasma centre resulting in a flattening of both the electron temperature and density profiles. In the case of on-axis heating, when the pre-crash electron temperature profile is sufficiently peaked, the formation of a hot ring around the sawtooth inversion surface is observed at the end of the reconnection process. When the ECH power is deposited slightly off-axis, the electron temperature profile is less peaked and the hot ring is not observed.

With ECH power deposited close to the sawtooth inversion surface, improved stability against the internal kink mode is obtained, leading to a long period of relative MHD quiescence and to pre-crash electron temperature profiles which are flat or even hollow inside the $q=1$ region. The subsequent sawtooth crash, observed during giant and humpback sawteeth, results in a small variation of the central electron temperature, which shows similar temporal evolution, despite a large difference in the electron density behaviour. This difference is not yet understood, but may be ascribed to the ECH associated pump-out. The post-crash electron temperature profiles in both sawtooth types are not poloidally symmetric, indicating an incomplete magnetic reconnection process.

The different types of sawteeth have been simulated using a numerical code based on a theoretical model [10] which describes the evolution of the electron temperature in the presence of localized heat sources and of an $m / n=1$ magnetic island whose temporal evolution is inferred from the experimental displacement function $\xi(t)$. Despite the relative simplicity of this model, the simulated sawtooth shapes under the different heating conditions are in agreement with the experimental observations.

\section{Acknowledgements}

The authors wish to thank K.A. Razumova for useful discussions and the entire TCV team for their help in this study. This work was partly supported by the Swiss National Science Foundation. F. Porcelli was supported in part by the Italian National Research Council (CNR). 


\section{Furno et al.}

\section{References}

[1] Lopez-Cardozo, N.J., et al., Phys. Rev. Lett. 73 (1994) 256.

[2] Cima, G., et al., Plasma Phys. Control. Fusion 40 (1998) 1149.

[3] Hofmann, F., et al., Plasma Phys. Control. Fusion 36 (1994) B277.

[4] Pietrzyk, Z.A., et al., Nucl. Fusion 39 (1999) 587.

[5] Razumova, K.A., et al., Plasma Phys. Rep. 23 (1997) 13.

[6] Goodman, T.P., et al., in Radiofrequency Heating and Current Drive of Fusion Devices (Proc. 2nd Eur. Top. Conf. Brussels, 1998), Vol. 22A, European Physical Society, Geneva (1998) 249.

[7] Pochelon, A., et al., Nucl. Fusion 39 (1999) 1807.

[8] Goodmann, T.P., et al., in International Congress on Plasma Physics (Proc. Congr. Prague, 1998), Vol. 22C, European Physical Society, Geneva (1998) 1324.

[9] Anton, M., et al., Plasma Phys. Control. Fusion 38 (1996) 1849.

[10] Porcelli, F., Rossi, E., Cima, G., Wootton, A., Phys. Rev. Lett. 82 (1999) 1458.

[11] Coppi, B., et al., Fiz. Plasmy 2 (1976) 961 [English translation: Sov. J. Plasma Phys. 1 (1976) 389].

[12] Dudok de Wit, T., Pecquet, A.L., Vallet, J.C., Lima, R., Phys. Plasmas 1 (1994) 3288.

[13] Snider, R.T., et al., Phys. Fluids B 1 (1989) 404.

[14] Hanada, K., et al., Phys. Rev. Lett. 66 (1991) 1974.

[15] Behn, R., et al., in Laser Aided Plasma Diagnostics (Proc. 7th Int. Symp. Fukuoka, 1995) 392.

[16] Smith, G., et al., in Electron Cyclotron Heating (Proc. 9th Joint Workshop Borrego Springs, 1995), World Scientific Publishing, Singapore (1995) 651.

[17] Goodman, T.P., et al., in Controlled Fusion and Plasma Physics (Proc. 26th Eur. Conf. Maastricht, 1999), Vol. 23J, European Physical Society, Geneva (1999) 1101.

[18] Anton, M., Dutch, M.J., Weisen, H., Rev. Sci. Instrum. 66 (1995) 3762.
[19] Weisen, H., et al., Nucl. Fusion 37 (1997) 1741.

[20] Weisen, H., Pasini, D., Weller, A., Edwards, A.W., Rev. Sci. Instrum. 62 (1991) 1531.

[21] Furno, I., Weisen, H., in Controlled Fusion and Plasma Physics (Proc. 27th Eur. Conf. Budapest, 2000), European Physical Society, Geneva (in press) paper P1-026.

[22] Angioni, C., Goodman, T.P., Pietrzyk, Z.A., Sauter, O., in Theory of Fusion Plasmas (Proc. Joint Varenna-Lausanne Int. Workshop, Varenna, 2000), Editrice Compositori, Bologna (2000).

[23] Boucher, D., et al., in Advances in Simulation and Modelling of Thermonuclear Plasmas (Proc. IAEA Tech. Comm. Mtg Montreal, 1993), IAEA, Vienna (1993) 142.

[24] Porcelli, F., Boucher, D., Rosenbluth, M.N., Plasma Phys. Control. Fusion 38 (1996) 2163.

[25] Wong, K.-L., et al., in Controlled Fusion and Plasma Physics (Proc. 27th Eur. Conf. Budapest, 2000), European Physical Society, Geneva (in press) paper P1-108.

[26] Coda, S., et al., in Controlled Fusion and Plasma Physics (Proc. 26th Eur. Conf. Maastricht, 1999), Vol. 23J, European Physical Society, Geneva (1999) 1097.

[27] Park, W., Monticello, D.A., Nucl. Fusion 30 (1990) 2413.

[28] Kadomtsev, B.B., Fiz. Plazmy 1 (1975) 710 [English translation: Sov. J. Plasma Phys. 1 (1976) 389].

[29] Lichtenberg, A.J., Nucl. Fusion 24 (1984) 1277.

[30] O'Rourke, J., Plasma Phys. Control. Fusion 33 (1991) 289.

[31] West, W.P., Thomas, D.M., de Grassie, J.S., Zheng, S.B., Phys. Rev. Lett. 58 (1987) 2758.

[32] Waelbroeck, F.L., Phys. Fluids B 1 (1989) 2372.

[33] Porcelli, F., et al., Nucl. Fusion 40 (2000) 1691.

(Manuscript received 17 May 2000

Final manuscript accepted 8 January 2001)

E-mail address of I. Furno: ivo.furno@epfl.ch

Subject classification: B0, Te; B0, Ti; G1, Te; G1, Ti 